\title{
Direct determination of the step-edge formation energies of the energetically stable and unstable double-layer step edges of $\mathrm{Si}(001)$
}

\author{
H. J. W. Zandvliet, S. van Dijken, and Bene Poelsema \\ Faculty of Applied Physics and Centre of Materials Research, University of Twente, P. O. Box 217, 7500 AE Enschede, The Netherlands
}

(Received 16 January 1996)

\begin{abstract}
Scanning tunneling microscopy images of $4.5^{\circ}$ misoriented double $B$ stepped $\mathrm{Si}(001)$ have been analyzed to determine the double-layer step-edge formation energies of the energetically stable double step ( $B$-type) as well as the energetically unstable double step ( $A$-type). The ordering of the various single- and double-layer step-edge formation energies is in accordance with semiempirical tight-binding-based total-energy calculations performed by Chadi [Phys. Rev. Lett. 59, 1691 (1987)]. Finally, the miscut angle at which the transition between the single- and double-layer stepped surface occurs as calculated using the experimentally obtained step-edge formation energies is in agreement with the experiments. [S0163-1829(96)05724-4]
\end{abstract}

One of the most intriguing and frequently studied surfaces is without any doubt $\mathrm{Si}(001)$. The $\mathrm{Si}(001)$ surface reconstructs to form rows of dimerized atoms. A rich variety of step structures have been reported in the literature over the past years. Among them are the transition from a hilly single-layer stepped surface to a surface containing coexisting areas with wavy single-layer steps and straight singlelayer steps, ${ }^{1}$ the transition from wavy and straight singlelayer steps to straight single-layer steps only ${ }^{2}$ and finally the transition from single-layer steps to double-layer steps. ${ }^{3} \mathrm{Be}$ cause of the symmetry of the diamond lattice, dimer rows are oriented in two perpendicular directions. Two types of single- and double-layer steps can be discriminated. Single(double-) layer step edges are denoted $S_{A}\left(D_{A}\right)$ when the dimer rows of the upper terrace run parallel to the step edge, whereas they are denoted $S_{B}\left(D_{B}\right)$ when the dimer rows run perpendicular to the step edge. From a technological point of view double-layer steps on $\mathrm{Si}(001)$ are particularly interesting, because the growth of, e.g., GaAs on single-layer stepped $\mathrm{Si}(001)$ gives rise to disorder in the overlayer, whereas double-layer steps allow the growth of coherent Ga and As layers. The first real-space images of double-layer steps on $\mathrm{Si}(001)$ were reported by Wierenga, Kubby, and Griffith. ${ }^{3}$ Their high-resolution scanning tunneling microscopy images have confirmed the rebonded geometry as proposed by Chadi. ${ }^{4}$ Besides the atomic structure of the various types of step edges of $\mathrm{Si}(001)$ Chadi also calculated their formation energies using a semiempirical tight-bindingbased total-energy calculation. The $S_{A}$ step edge turns out to have the lowest formation energy because it is the only step edge that does not lead to large strains or to extra dangling bonds. The $D_{A}$ step edge has the highest formation energy. Scanning tunneling microscopy images of single-layer stepped $\mathrm{Si}(001)$ have been analyzed to determine the stepedge formation energies directly. From the roughness of the $S_{B}$ the formation energy of the $S_{A}$ can be extracted and vice versa. ${ }^{5,6}$ On double-layer stepped $\mathrm{Si}(001)$ only $D_{B}$-type steps occur. Determination of both $D_{A}$ and $D_{B}$ step-edge formation energies seems therefore a priori impossible. However, in this paper we will show that from the roughness of the $D_{B}$ step edge alone both double-layer step-edge formation energies can be determined. Eaglesham et al. ${ }^{7}$ measured the equilibrium shape of voids in Si formed by $\mathrm{MeV}$ He implantation and annealing. From this shape they were able to extract the surface energy, $\gamma(\theta)$. The step-edge formation energy for the $D_{B}$ step edge obtained from $d \gamma / d \theta$ was determined to be $92 \mathrm{meV} / 2 \mathrm{a}$. This value is in good agreement with the earlier mentioned theoretical calculation (100 meV/2a) by Chadi. ${ }^{4}$ To the best of our knowledge the $D_{A}$ step-edge formation energy has not been determined experimentally.

All experiments were carried in an ultrahigh-vacuum (UHV) chamber with a base pressure in the mid$10^{-11}$-Torr range. The chamber, which is mounted on pneumatic legs, contains a home-built STM (scanning tunneling microscope). The $\mathrm{B}$-doped $p$-type $\mathrm{Si}(001)$ samples $(5-10$ $\Omega \mathrm{cm}$ ) with dimensions $20 \times 4 \times 0.3 \mathrm{~mm}^{3}$ were $4.5^{\circ}$ misoriented toward [110]. The azimuthal misalignment of the step edge with respect to the $[\overline{1} 10]$ direction was estimated to be less than $0.5^{\circ}$. After loading the samples in the UHV chamber they were outgassed at a temperature of about 875-975 $\mathrm{K}$ for at least $20 \mathrm{~h}$ followed by flashing at temperatures above $1575 \mathrm{~K}$ for several seconds. After flashing the samples were either quenched immediately to room temperature by turning off the power supply or quenched to room temperature in a two-step process: first to a temperature varying between 600 and $825 \mathrm{~K}$, kept there for about $15 \mathrm{~min}$, followed by a second quench to room temperature. It turns out that, in agreement with previous work, ${ }^{6}$ the different cooling procedures have no measurable influence on the observed stepedge roughness and kink distribution. During flashing the chamber pressure was kept in the $10^{-10}$-Torr range. This procedure results in clean nearly single domain $\mathrm{Si}(001)$ $2 \times 1$ surfaces with a defect density as low as $0.5 \%$.

In this paper, we present scanning tunneling microscopy measurements of the step-edge roughness of the $D_{B}$ step that allows a determination of the step-edge formation energies of both types of double-layer step edges. In Figs. 1(a) and 1(b) two filled-state STM images of $4.5^{\circ}$ misoriented $\mathrm{Si}(001)$ are displayed. Areas with rebonded $D_{B}$ double steps coexist with areas where the double step has split into pairs of singles $\left(S_{B}+S_{A}\right)$. To determine the distribution of step con- 


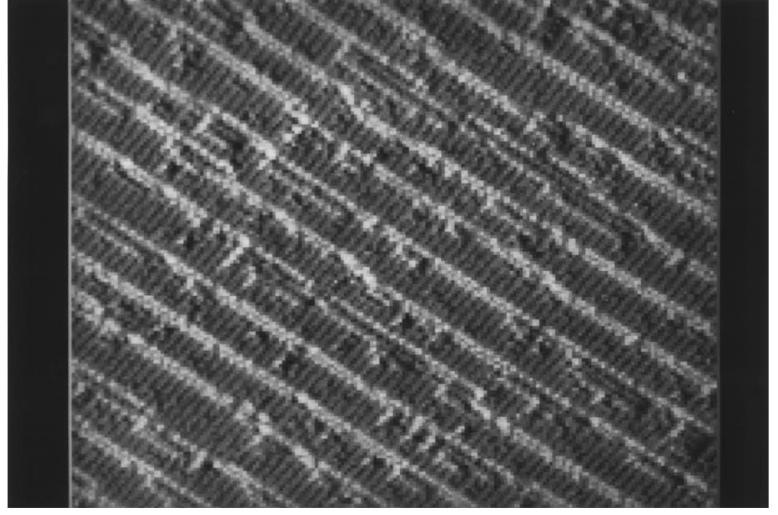

(a)

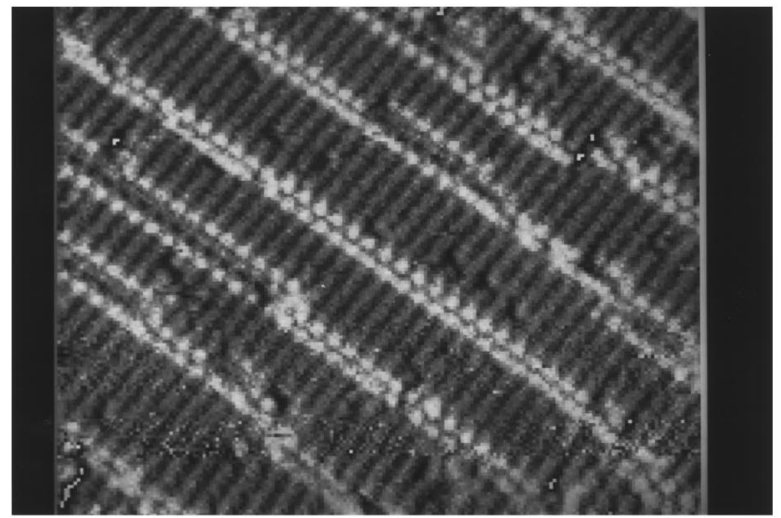

(b)

FIG. 1. Scanning tunneling microscopy images of $4.5^{\circ}$ vicinal $\mathrm{Si}(001)$ tilted toward [110]. Sample bias $-2 \mathrm{~V}$ and tunneling current $1 \mathrm{nA}$. (a) Scan size $40 \times 32 \mathrm{~nm}^{2}$; (b) scan size $20 \times 16 \mathrm{~nm}^{2}$.

figurations, we have measured the probability of finding various step-edge geometries. We have, however, only counted several types of different step-edge geometries [see Figs. 2(a) -2(c)]: the rebonded $D_{B}$ step geometry (denoted $n_{D_{B}}$ ), a combination of two single-layer step edges separated by $2 a$ $(=7.7 \AA)$ from each other (denoted $n_{S_{A}+S_{B}}$ ), and finally kinks with length $2 a$ consisting of a $D_{A}$ piece of step edge (denoted $n_{D_{A}}$ ). Under the assumption of independent kinks and no azimuthal misalignment of the step edge with respect to the $[\overline{1} 10]$ direction (no forced kinks), the probability of finding a certain type of kink is $\propto g_{i} \exp [-E(n) / k T]$, where $T$ is the freeze in temperature of the step-edge roughness [estimated to be about $775 \mathrm{~K}$ (Ref. 6)], $E(n)$ is the energy difference between the kink position under consideration and a rebonded $D_{B}$ position (our reference), and $g_{i}$ arises from the degeneracy of the excited states; i.e., there are in general two possible kink directions, either into or out of the terrace. For the $n_{D_{A}}$ type of kink the two possible kink directions occur with the same probability, indicative of no azimuthal misalignment of the step edge. The difference in energy between a rebonded $D_{B}$ step edge and an $n_{D_{A}}$ type of kink of length $2 a(=7.7 \AA)$ is just the formation energy of a $D_{A}$ step edge per $2 a$

$$
n_{D_{A}} / n_{D_{B}}=2 e^{-E_{D_{A}} / k T}
$$

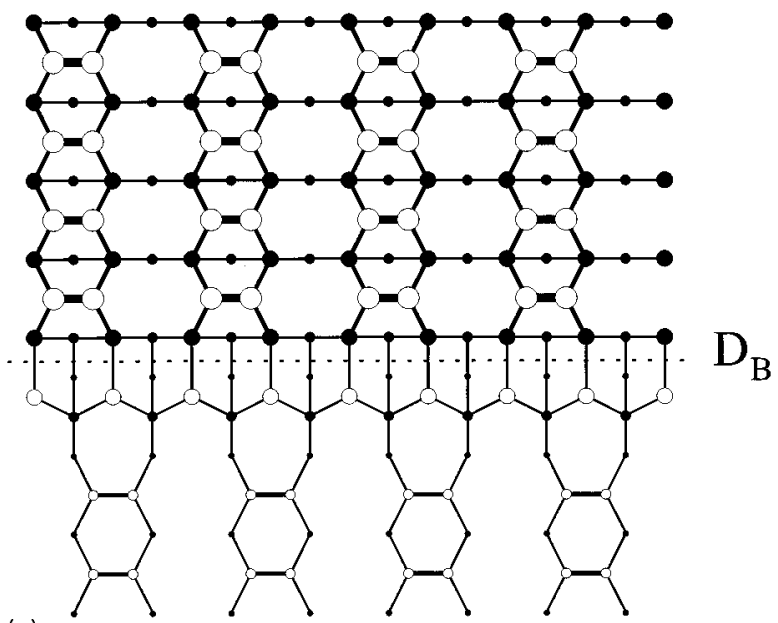

(a)

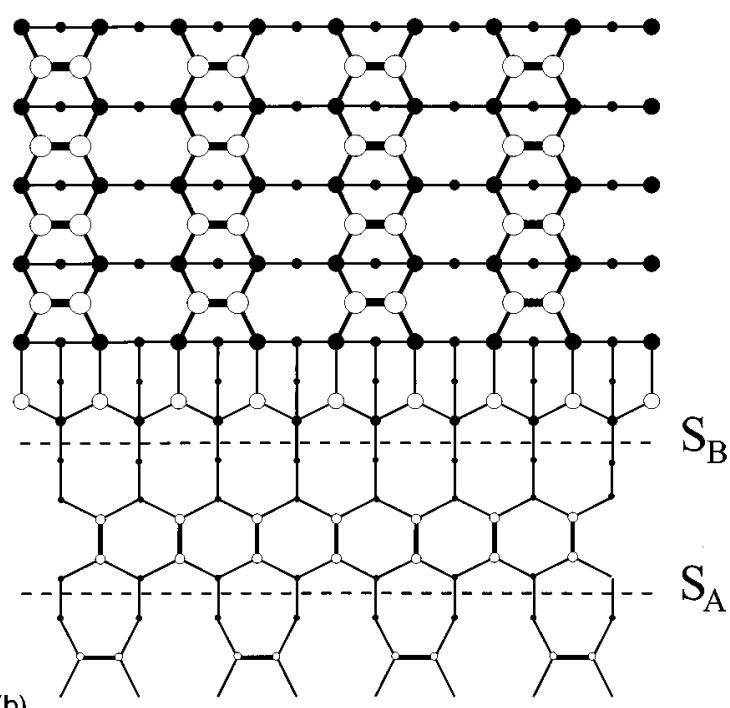

(b)

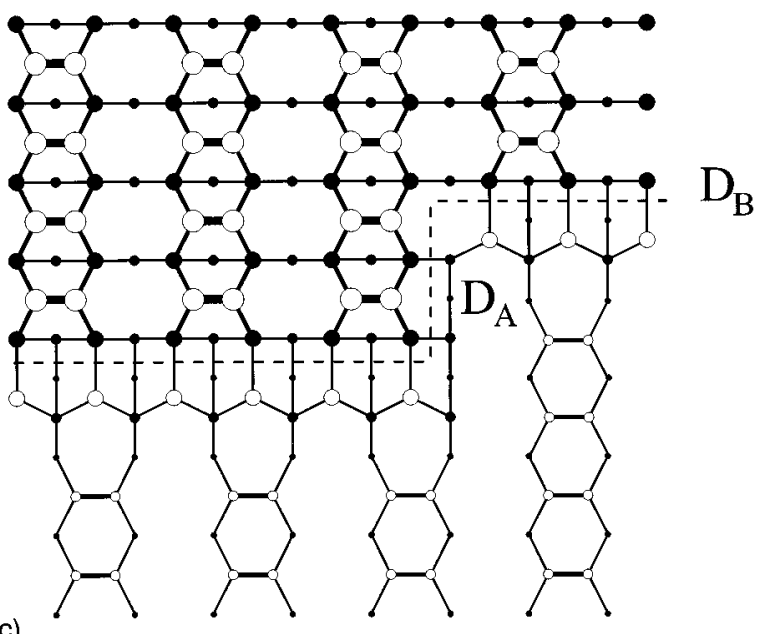

FIG. 2. Configuration of various step geometries. (a) $n_{D_{B}}$ stepedge geometry; (b) $n_{S_{A}+S_{B}}$ step-edge geometry; (c) $n_{D_{A}}$ kink position.

We have counted in total more than 2000 step-edge positions $\left(n_{D_{B}}=1521, n_{D_{A}}=33\right.$, and $\left.n_{S_{A}+S_{B}}=472\right)$. From these numbers we extract a ratio $n_{D_{A}} / n_{D_{B}}$ of 0.022 resulting in a $D_{A}$ step-edge formation energy of $300 \mathrm{meV} / 2 a$. As we will see 
below the $D_{A}$ step-edge formation energy is substantially higher than the formation energy of a $D_{B}$ type of step edge in agreement with the experiments that no $D_{A}$ step edges are observed after cleaning $\mathrm{Si}(001)$. In order to extract the $D_{B}$ step-edge formation energy we focus our attention on the probability of occurrence of a step-edge geometry where the two single-layer step edges are separated by $2 a$. The difference in energy between a rebonded $D_{B}$ step edge and this combination of two single-layer step edges separated by $2 a$ is $E_{S_{A}}+E_{S_{B}}+E_{\text {strain }}-E_{D_{B}}$ (Refs. 8 and 9)

$$
n_{S_{A}+S_{B}} / n_{D_{B}}=e^{-\left(E_{S_{A}}+E_{S_{B}}+E_{\text {strain }}-E_{D_{B}}\right) / k T} .
$$

From the experiments we extract a ratio $n_{S_{A}+S_{B}} / n_{D_{B}}$ of 0.31 . The strain relaxation energy per unit step length, $E_{\text {strain }}$, can be written as ${ }^{8}$

$$
E_{\text {strain }}=-2 C \ln \left[\frac{l+L}{2 \pi a} \sin \left(\frac{\pi l}{l+L}\right)\right],
$$

where $l(=7.7 \AA)$ is the width of the minority domain and $L\left(=\left[2.72 \AA / \tan \left(4.5^{\circ}\right)\right]-7.7 \AA=26.9 \AA\right)$ the width of the majority domain. $C$ is a function of the elastic constants and of the difference of the surface stress within the two types of $2 \times 1$ domains and $a_{0}$ is a microscopic cutoff length (e.g., the lattice constant). ${ }^{8}$ From various experiments $C$ is determined to be about $25 \mathrm{meV} / 2 a,{ }^{9,10}$ resulting in a strain relaxation energy of only $4 \mathrm{meV} / 2 a$. The step-edge formation energies of the single-layer steps have been determined earlier ${ }^{5,6}$ to be 120 and $52 \mathrm{meV} / 2 a$ for the $S_{B}$ and $S_{A}$ step edges, respectively. Plugging these values into Eq. (2) gives a step-edge formation energy of $100 \mathrm{meV} / 2 a$ for the $D_{B}$ step edge. This experimentally determined $D_{B}$ step-edge formation energy is in good agreement with the experimental value reported by Eaglesham et al. ${ }^{7}$ as well as the theoretical calculation by Chadi. ${ }^{4}$

In order to give an estimate of the critical angle at which the transition between the single-layer stepped surface and the double-layer stepped $\mathrm{Si}(001)$ surface one should realize that the double-layer stepped $\mathrm{Si}(001)$ surface is a singledomain structure where all the terraces have the same orientation, and no strain relaxation can occur. On the other hand, a single-layer stepped $\mathrm{Si}(001)$ surface exhibits two different terraces, whose orientation alternates. The corresponding stress tensor (the $2 \times 1$ reconstruction has an anisotropic stress tensor) rotates at each single-layer step edge, resulting in a strain relaxation energy (per unit area) for the singlelayer stepped surface with an averaged terrace width $\lambda$ of $C \ln (\lambda / \pi a)$. With decreasing miscut angle the strain relaxation becomes large enough to make a combination of two single-layer steps (formation energy $172 \mathrm{meV} / 2 a$ ) energetically more favorable as compared to a single double-layer step (formation energy $100 \mathrm{meV} / 2 a$ ). The miscut angle, $\theta_{c}$, where the transition occurs from a single-layer stepped to a double-layer stepped $\mathrm{Si}(001)$ surface can be extracted from the following equation:

$$
E_{D_{B}}=E_{S_{A}}+E_{S_{B}}-2 C \ln \left(\lambda_{c} / \pi a\right),
$$

where $\lambda_{c}\left[=2.72 \AA / \tan \left(\theta_{c}\right)\right]$ is the critical terrace width. Plugging the values for $E_{D_{B}, S_{A}, S_{B}}(100,52$, and $120 \mathrm{meV} /$ $2 a$ ) and $C(25 \mathrm{meV} / 2 a)$ in Eq. (4) results in a critical miscut angle $\theta_{c}$ of $1.5^{\circ}$ in good agreement with the experimental observations. Because thermal fluctuations of the step edges have not been taken into account here this result only holds for $T=0 \mathrm{~K}$. The entropy of the two wandering single-layer steps is larger as compared to the entropy of the wandering double step resulting in a decrease of the critical terrace width $\lambda_{c}$ and thus an increase of the critical angle $\theta_{c}$.

In summary, scanning tunneling images of double-layer stepped $\mathrm{Si}(001)$ are analyzed in order to determine the stepedge formation energies of the two types of double-layer step edges. The $D_{B}$ step-edge formation energy is estimated to be about $100 \mathrm{meV} / 2 a$, whereas the energetically unfavorable $D_{A}$ step edge has a formation energy of about $300 \mathrm{meV} /$ $2 a$. The $D_{B}$ step-edge formation energy is in good agreement with the experimental value of Eaglesham et al. ${ }^{7}$ as well as the theoretical calculation by Chadi. ${ }^{4}$ Moreover, the ordering of the two single- and two double-layer step-edge formation energies is in accordance with the ordering reported by Chadi. ${ }^{4}$ Finally, using these values of the step-edge formation energies as well as a value of the strain relaxation energy of the $2 \times 1$ domains we are able to estimate quite accurately the miscut angle for which the transition between single stepped $\mathrm{Si}(001)$ to double-layer stepped $\mathrm{Si}(001)$ occurs. The calculated miscut angle of $1.5^{\circ}$ is in agreement with the experimental observations.
${ }^{1}$ R. M. Tromp and M. C. Reuter, Phys. Rev. B 47, 7598 (1993).

${ }^{2}$ R. M. Tromp and M. C. Reuter, Phys. Rev. Lett. 68, 820 (1992).

${ }^{3}$ P. E. Wierenga, J. A. Kubby, and J. E. Griffith, Phys. Rev. Lett. 59, 2169 (1987).

${ }^{4}$ D. J. Chadi, Phys. Rev. Lett. 59, 1691 (1987).

${ }^{5}$ B. S. Swartzentruber et al., Phys. Rev. Lett. 59, 1913 (1990).

${ }^{6}$ H. J. W. Zandvliet et al., Phys. Rev. B 45, 5965 (1992).
${ }^{7}$ D. J. Eaglesham et al., Phys. Rev. Lett. 70, 1643 (1993); 70, 1392(E) (1994); 70, 2975 (1994).

${ }^{8}$ P. Zeppenfeld et al., Phys. Rev. Lett. 72, 2737 (1994).

${ }^{9}$ H. J. W. Zandvliet and H. B. Elswijk, Phys. Rev. B 48, 14269 (1993).

${ }^{10}$ F. Wu and M. G. Lagally, Phys. Rev. Lett. 75, 2534 (1995). 